\title{
Energy and $\mathrm{CO}_{2}$ efficient scheduling of smart appliances in active houses equipped with batteries
}

\author{
Kaveh Paridari, Alessandra Parisio, Henrik Sandberg and Karl Henrik Johansson
}

\begin{abstract}
In this paper, we present a novel method for scheduling smart appliances and batteries, in order to reduce both the electricity bill and the $\mathrm{CO}_{2}$ emissions. Mathematically, the scheduling problem is posed as a multi-objective Mixed Integer Linear Programming (MILP), which can be solved by using standard algorithms. A case study is performed to assess the performance of the proposed scheduling framework. Numerical results show that the new formulation can decrease both the $\mathrm{CO}_{2}$ emissions and the electricity bill. Furthermore, a survey of studies that deal with scheduling of smart appliances is provided. These papers use methods based on MILP, Dynamic Programming (DP), and Minimum Cut Algorithm (MCA) for solving the scheduling problem. We discuss their performance in terms of computation time and optimality versus time discretization and number of appliances.
\end{abstract}

\section{INTRODUCTION}

The need to satisfy the increasing energy demand in a sustainable way requires active energy distribution networks, i.e., distribution networks with the possibility of bidirectional power flows controlling a combination of distributed energy resources, such as storage devices and renewable energy sources. Hence, distribution companies are required to consider more complex scenarios due to the extra stress created to balance energy demand and generation. Load balancing must be accomplished so that the quality of electrical supply to consumers is maintained and the usage of non-renewable generation is minimized.

In this scenario, Demand Response (DR) has received increased attention in recent years since it can efficiently support load balancing and economical/environmental cost reduction [1], [2]. DR is commonly defined as changes in electricity use by consumers in response to changes in the electricity price over time [1]; further, the electricity use can also be sensitive to changes in the $\mathrm{CO}_{2}$ emission. DR policies aim at flattening the demand curve, which helps preventing grid instability and reduce line losses, as well as the number of expensive generation plants used only for peak load periods. Naturally, effective DR policies require smart appliances, which can be switched on or off in response to price and $\mathrm{CO}_{2}$ signals.

Several studies have investigated the potential changes in residential electricity use under time-varying price rates by

All the authors are with the ACCESS Linnaeus Center and the Automatic Control Lab, School of Electrical Engineering, KTH Royal Institute of Technology, Sweden. Emails: paridari@kth.se, parisio@kth.se, hsan@kth.se and kallejekth.se

This work is supported by the European Institute of Technology (EIT) Information and Communication Technology (ICT) Labs, the Swedish Energy Agency, Swedish Foundation of Strategic Research through the ICTPsi project, the Swedish Governmental Agency for Innovation Systems (VINNOVA) and the Knut and Alice Wallenberg Foundation. rescheduling smart appliances, e.g., see [3], [4], [5]. Other works have also focused on $\mathrm{CO}_{2}$ emission factors and its potential impacts on the changes in household load profile, e.g., see [6]. The main conclusions are that employing hourly electricity prices and DR technologies can be highly beneficial for the distribution grid and compel consumers to arrange an economical/environmental way of using smart appliances. Further, another important conclusion is that the success of DR programs depends on the awareness and behavioral adaption of consumers. It is clearly too hard for consumers to reschedule their appliances based on time-varying electricity prices and $\mathrm{CO}_{2}$ emission, keep monitoring these signals over the whole day and, additionally, conveniently use the storage device [7], [8]. Hence, automated decision support systems are needed to optimally schedule household appliances.

Further, by integrating batteries with DR policies, it would be more flexible and efficient for consumers to manage their energy use in response to time-varying electricity prices and network congestion, taking advantage of the capability of these devices to store energy and release it when it is more convenient. Studying the impact of DR policies that employ storage devices is motivated by the cost reduction of small-scale devices, by the technological advances and the availability of physical infrastructures for accommodating energy storage devices.

Several works have proposed load management strategies accounting for price and $\mathrm{CO}_{2}$ information (e.g., see [1], [9], [10], [11], [12], [13], [14], [15] and the Stockholm Royal Seaport project [16]). The aforementioned works do not consider batteries, which are taken into account in [17], [18].

Differently from the aforementioned works, in this paper we aim to model the decision problem as realistically as possible, accounting, for instance, for energy degradation and power losses in batteries including different multiplicative factor in charging and discharging. Hence, simplified analytical and linear-programming based analysis tools, as in [15], [17], are not employed. Instead, in this work we take advantage of the modeling capabilities and the computational advances of MILP algorithms for stating a residential appliance scheduling problem that aims at optimizing a trade off between electricity costs and $\mathrm{CO}_{2}$ emission (in certain countries including Sweden [12]) by purchasing energy from the grid and/or storing energy to use it when more beneficial. Further, we account for user time preferences and the possibility to sell stored energy to the grid.

This paper conducts a numerical study to investigate, in a realistic test case, the impact of DR policies that consider both 


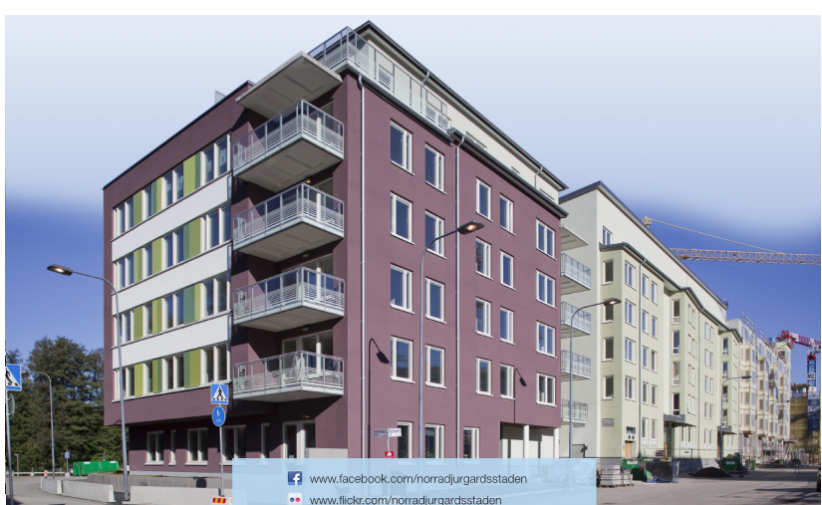

Fig. 1. An active apartment building in Royal Seaport project in Stockholm.

electricity price and $\mathrm{CO}_{2}$ signals as well as batteries. Further, we compare three commonly employed methods in the literature to solve the appliance scheduling problem without batteries: $i$ ) a MILP formulation proposed in [11], solved by branch and bound techniques; ii) a discrete-time formulation solved by the minimum cut algorithm, as proposed in [13]; iii) a dynamic programming based procedure proposed in [12]. Advantages and limitations of these methods are then discussed.

The paper is organized as follows. Section II describes the realistic scenario under study. Section III is devoted to formulate the scheduling problem of smart appliances and battery, and discusses numerical results. Section IV presents a survey on different methods for scheduling smart home appliances and discusses their performance. Finally, Section V provides conclusions and future studies.

\section{AN ACtive house In the Royal SEAPORT PRoJect}

In our case study we consider active houses, which are houses where effective DR policies are enabled through the integration of smart appliances, scheduling algorithms, energy management systems, and information exchange over wireless communication technologies. Within the Stockholm Royal Seaport project [16], which is a new, environmentally sustainable city district being built in Stockholm, some active apartment buildings are available and occupied by families; an example of an active apartment building is depicted in Figure 1.

We consider a case study of 10000 active apartments with smart appliances, for the evaluation of DR programs, and to predict the potential load shift and bill and $\mathrm{CO}_{2}$ savings of households. Actual hourly power consumption profiles from active apartments in the Stockholm Royal Seaport are used in this work. The data has been kindly provided by Fortum Corporation, which is actively involved in the project.

\section{A. Power consumption of an active house}

In this paper, we want to investigate the potential of active houses for saving electricity bill and reducing $\mathrm{CO}_{2}$ emission by using an automation system for scheduling of smart appliances and batteries. Therefore, for assessment of the automation system, it is necessary to have the information related to hourly energy consumption in active houses without

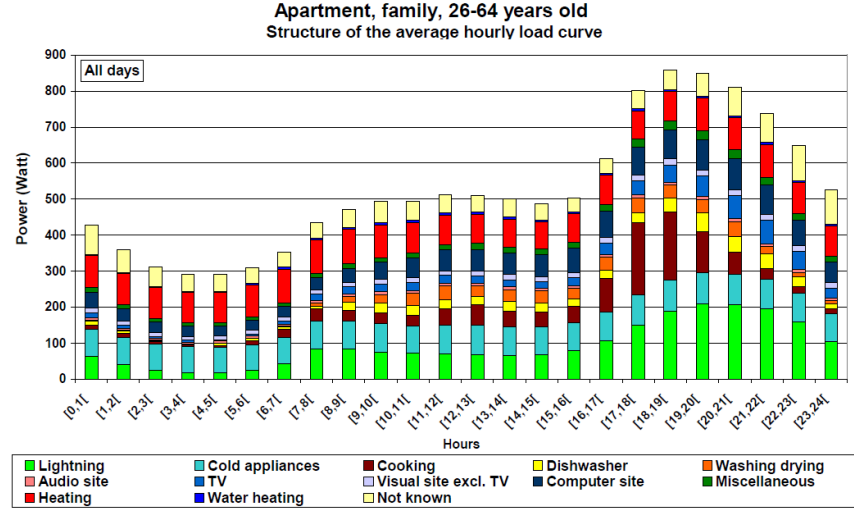

Fig. 2. Average hourly power consumption for years 2005-2008 [14]. automation system, and the portion of household appliances in this energy consumption. In the Stockholm Royal Seaport project, two active apartments are occupied by families and hourly power consumption is available from March 2013 to January 2014. To determine the hourly power consumption of household appliances vs other consumptions, a comparison with previous works is done. In [14], apartments average hourly power consumption is a result of the empirical measuring of a total of 199 apartments between the years 2005-2008 in Sweden, which is done by the Swedish Energy Agency. In that study, five types of apartments (singles 26-64 years old and above the age of 64, couples 26-64 years old and above the age of 64 and families 26-64 years old) are taken into account. Among all these 199 apartments, 125 of them were occupied by families 26-64 years old (more than 60\%) and in this paper the data related to them is used. Fig. 2 shows average hourly power consumption of apartments occupied with families who are 26-64 years old, in which $4 \%$ of the consumption is devoted to run washing-machine and dryer and $4 \%$ for dish-washer, and the remaining consumption is used for the other appliances.

Based on these information and considering Fig. 2, estimated average hourly power consumption of appliances vs other consumptions for the two active apartments is shown in Fig. 3. As it is obvious in these two figures, the average hourly load curve profile of the studied active apartments is very similar to that of the apartments monitored in [14]. The only thing to be noticed, is that the total amount of energy being used in one day in the active apartments is approximately 9.9 $\mathrm{kWh}$ on average, and in comparison with the apartments that were studied in [14] (consuming 12,6 kWh on average) has decreased more than $20 \%$. This reduction in power consumption is reasonable based on the modern home appliances that are used in the active apartments.

\section{OPTIMAL SCHEDULING OF SMART APPLIANCES AND BATTERIES IN ACTIVE HOUSES}

The aim of scheduling smart appliances in active houses is to reduce the electricity bill and $\mathrm{CO}_{2}$ emission, and in many situations there exist a conflict between electricity bill and $\mathrm{CO}_{2}$ emission to be minimized at the same time [12]. To achieve the goal of optimal scheduling, different methods have been proposed to deal with this possible conflict and 


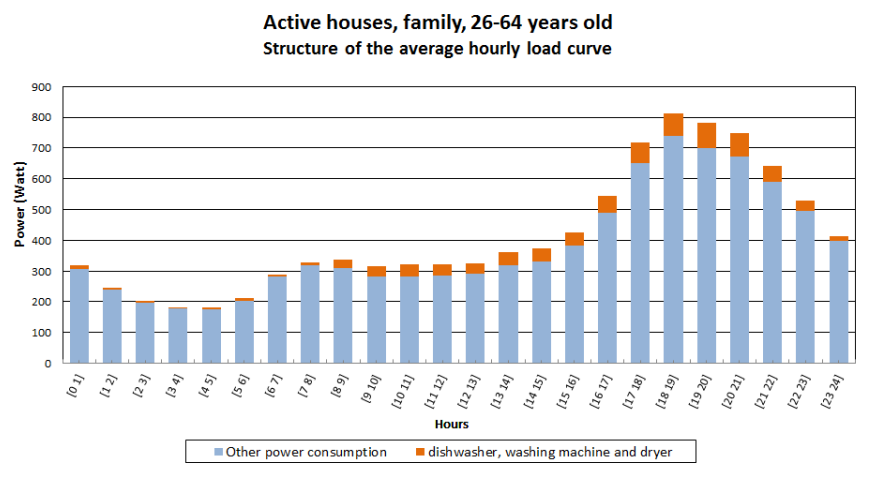

Fig. 3. Estimated average hourly power consumption of appliances vs other consumptions (for March 2013 - January 2014) of two active apartments.

trade-off. Weighted sum and $\varepsilon$-constrained approaches are two of these methods that have mostly been used in the literature [13], [12], [11]. So the problem is a multi-objective minimization of electricity bill and $\mathrm{CO}_{2}$ emission, and there exist constraints on the user preferences and operation process of appliances. That means we are concerned with the scheduling of a number of user-specified home-appliances in a certain period of time, in which the user can also specify precedence relations between certain appliances (user preferences). In this scheduling framework, the operation process of an appliance is divided into a set of sub-tasks (energy phases) of the appliance operation (i.e. movement, pre-heating, heating, etc for washing machine [11]) and it is considered that, once an energy phase starts, it must continue until it is finished. In addition, there exists a flexible delay between energy phases of each appliance that is modeled by a minimum and maximum delay time (constraints on the operation process of appliances). Based on our knowledge till now, the mixed integer linear programming (MILP) framework that was proposed in [11] is more extendable (e.g. for including batteries as dynamical systems) than the other methods proposed in the literature. Therefore, in this paper we formulate the smart home appliances scheduling problem (with and without battery) by extending the MILP framework in [11], which considers the minimum electricity cost and satisfies technical operation constraints, consumer preferences and peak power consumption. In this paper, electricity tariff and $\mathrm{CO}_{2}$ footprint signals are assumed to be piecewise constant, and the MILP scheduling problem is solved using CPLEX (using the YALMIP MATLAB interface [19]), which is a commercial implementation of a branch-andbound algorithm.

\section{A. Scheduling appliances without using battery}

In the mathematical formulation for scheduling of smart appliances in [11], the appliances execution period is discretized into $m$ uniform time slots (e.g. 10 minutes per slot). The number of appliances considered for scheduling is denoted by $N$, and $n_{i}$ for $i=1,2, \ldots, N$, denotes the number of uninterruptible energy phases for each appliance. The energy assigned to energy phase $j$ of appliance $i$ during the whole period of time slot $k$ is denoted by $p_{i j}^{k}$. In addition, auxiliary binary decision variables $\left(x_{i j}^{k}\right)$ are required to indicate whether a particular energy phase is being processed or not. Moreover, two other sets of binary decision variables are needed to model the decision problem. One is denoted as $s_{i j}^{k}$, with a value of one indicating that, in appliance $i$, energy phase $j$ is already finished by time slot $k$. The other set is denoted as $t_{i j}^{k}$. These decision variables are used to indicate whether at time slot $k$, appliance $i$ is making a transition between running phase $j-1$ to $j$. We formulate a multiobjective optimization problem in which the cost function is parameterized with the weighting parameter $\lambda \in[0,1]$ (that would be chosen by end-users) as follow

$$
\sum_{k=1}^{m}\left((1-\lambda) E^{k}+\lambda C^{k}\right)\left(\sum_{i=1}^{N} \sum_{j=1}^{n_{i}} p_{i j}^{k}\right) .
$$

To generate the Pareto curve [20], considering the electricity bill and $\mathrm{CO}_{2}$ emission, following normalizations are applied

$$
E^{k}=\frac{e^{k}}{\max \left(e^{1}, e^{2}, \ldots, e^{m}\right)}, \quad C^{k}=\frac{c^{k}}{\max \left(c^{1}, c^{2}, \ldots, c^{m}\right)},
$$

where $e^{k}$ and $c^{k}$ denote the electricity bill and $\mathrm{CO}_{2}$ footprint for time slot $k$ respectively and based on given 24-hour ahead tariff curves (which are piecewise constant). Note that $\lambda=0$ implies end-users to only care about the electricity bill, while for $\lambda=1$ they only take $\mathrm{CO}_{2}$ emission into account. The optimization problem in (1) is solved based on defined variables and subject to the following constraints.

The constraint that is enforced to make sure that the energy phases fulfill their energy requirement is as

$$
\sum_{k=1}^{m} p_{i j}^{k}=E R_{i j}, \quad \forall i, j,
$$

where $E R_{i j}$ is the energy requirements for energy phase $j$ in appliance $i$. In addition, to determine that an energy phase is being processed during time slot $k$, while the limitation on lower and upper power assignment to the phase are satisfied, the constraint

$$
\underline{p}_{i j}^{k} x_{i j}^{k} \leq p_{i j}^{k} \leq \bar{p}_{i j}^{k} x_{i j}^{k}, \quad \forall i, j, k,
$$

is enforced. Also, the power safety constraint can be imposed as

$$
\sum_{i=1}^{N} \sum_{j=1}^{n_{i}} p_{i j}^{k} \leq \bar{P}^{k}, \quad \forall k
$$

where $\bar{P}^{k}$ is the upper limit of the total energy assigned at time slot $k$.

The limits on energy phases process time are imposed as

$$
\underline{T}_{i j} \leq \sum_{k=1}^{m} x_{i j}^{k} \leq \bar{T}_{i j}, \quad \forall i, j .
$$

To satisfy the sequential processing of the energy phases of an appliance and also sequential operation between appliances, the following constraints are imposed respectively

$$
\begin{array}{ccc}
x_{i j}^{k} \leq s_{i(j-1)}^{k}, & \forall i, k, \forall j=2, \ldots, n_{i}, \\
x_{i j}^{k} \leq s_{\tilde{i} n_{\tilde{i}}}^{k}, & \forall k,
\end{array}
$$


where $\underline{T}_{i j}$ and $\bar{T}_{i j}$ are the lower and upper limits of the number of time slots for energy phase $j$ in appliance $i$ to be processed, and $\tilde{i}$ being the index of the appliance which must be finished before $i$ can start. To make sure that energy phases are uninterruptible the following constraint is imposed.

$$
\begin{array}{ccc}
x_{i j}^{k} & \leq 1-s_{i j}^{k} & \forall i, j, k \\
x_{i j}^{k-1}-x_{i j}^{k} & \leq s_{i j}^{k} & \forall i, j, \forall k=2, \ldots, m \\
s_{i j}^{k-1} & \leq s_{i j}^{k} & \forall i, j, \forall k=2, \ldots, m .
\end{array}
$$

To count the number of time slots spent between the energy phases in an appliance and impose lower and upper limits on this number, the constraints

$$
\begin{gathered}
t_{i j}^{k}=s_{i(j-1)}^{k}-\left(x_{i j}^{k}+s_{i j}^{k}\right) \forall i, j, \forall k=2, \ldots, n_{i}, \\
\underline{D}_{i j} \leq \sum_{k=1}^{m} t_{i j}^{k} \leq \bar{D}_{i j}, \forall i, \forall j=2, \ldots, n_{i},
\end{gathered}
$$

are considered, where $\underline{D}_{i j}$ and $\bar{D}_{i j}$ are between-phase delay lower and upper bounds, respectively. Finally, to meet the household preferences and finishing a particular appliance within a specified time interval, the constraint

$$
x_{i j}^{k} \leq T P_{i}^{k} \quad \forall i, j, k,
$$

is enforced, where $T P_{i}^{k}$ characterizes the time preference interval. To summarize, the complete minimization problem (1) can be written as

$$
\begin{array}{cl}
\underset{p, x, s, t}{\operatorname{minimize}} & \sum_{k=1}^{m}\left((1-\lambda) E^{k}+\lambda C^{k}\right)\left(\sum_{i=1}^{N} \sum_{j=1}^{n_{i}} p_{i j}^{k}\right), \lambda \in[0,1] \\
\text { subject to } & \text { constraints }(3)-(11) \\
& p_{i j}^{k} \in \mathbb{R}, \quad \forall i, j, k \\
& x_{i j}^{k}, s_{i j}^{k} \in 0,1, \quad \forall i, j, k \\
& t_{i j}^{k} \in 0,1, \quad \forall i, k, \quad \forall j=2, \ldots, n_{i} .
\end{array}
$$

\section{B. Scheduling appliances by using battery}

To investigate battery usage effects on saving electricity bill and $\mathrm{CO}_{2}$ emission, battery can be modeled as a set of constraints and be included in the minimization problem (12). This battery formulation is an extension of the formulation in [21] by including the limits on the number of charging and discharging cycles. The level of energy stored in the battery at time slot $k$, should always satisfies the lower $\left(\underline{b}_{s}\right)$ and upper $\left(\bar{b}_{s}\right)$ limitations

$$
\underline{b}_{s} \leq b_{s}^{k} \leq \bar{b}_{s}, \quad \forall k,
$$

where $b_{s}^{k}$ is the state of charge $(S O C)$ of battery in time slot $k$. Moreover, to meet the lower and upper limitations on power exchanged with battery when it is charging or discharging during time slot $k$, the two constraints

$$
0 \leq b_{c}^{k} \leq \bar{b}_{c}^{k} x_{c}^{k}, \quad 0 \leq b_{d}^{k} \leq \bar{b}_{d}^{k} x_{d}^{k}, \quad \forall k
$$

are enforced, in which the auxiliary binary decision variables $x_{c}^{k}$ and $x_{d}^{k}$ indicate whether the battery is charging or discharging in time slot $k$, respectively. The power exchanged with battery during time slot $k$ is denoted by $b_{c}^{k}$ (or $b_{d}^{k}$ ) when the battery is charging (or discharging). In addition, the constraint

$$
x_{c}^{k}+x_{d}^{k} \leq 1, \quad \forall k,
$$

should be satisfied to make sure that the battery is not charging and discharging at the same time slot. To take the state of health of batteries into account, the total number of charging and discharging cycles during a day should be limited to a determined number $N_{c}$, and the constraints

$$
\begin{aligned}
& x_{c}^{k}-x_{c}^{k-1} \leq c_{t}^{k}, \quad \forall k=2, \ldots, m \\
& x_{d}^{k}-x_{d}^{k-1} \leq d_{t}^{k}, \quad \forall k=2, \ldots, m \\
& \sum_{i=1}^{m} c_{t}^{k}+d_{t}^{k} \leq N_{c},
\end{aligned}
$$

should be satisfied, where the auxiliary binary decision variables $c_{t}^{k}$ and $d_{t}^{k}$ determine the transition time slots to start charging and discharging, respectively. The dynamic system constraint

$$
b_{s}^{k}=\alpha b_{s}^{k-1}+\eta_{c} b_{c}^{k-1}-\eta_{d} b_{d}^{k-1}, \quad \forall k=2, \ldots, m,
$$

describes the evolution of energy stored in the battery, in which the $\alpha$ is a constant stored energy degradation in each sampling interval, and $\eta_{c}$ and $\eta_{d}$ are efficiencies accounting for the losses during charging and discharging. To satisfy the power balance in the system, the constraint

$$
\sum_{i=1}^{N} \sum_{j=1}^{n_{i}} p_{i j}^{k}+b_{c}^{k}-b_{d}^{k}=p_{G}^{k}, \quad \forall k,
$$

is enforced, where the exchanged power with the grid is denoted by $p_{G}^{k}$, and it should satisfy lower and upper limitations.

$$
\underline{p}_{G}^{k} \leq p_{G}^{k} \leq \bar{p}_{G}^{k}, \quad \forall k,
$$

where the lower limit is negative to allow energy selling to the grid. Finally, it is reasonable to assume that the initial and the final energy levels ( $b_{s}^{0}$ and $b_{s}^{T}$ respectively) in the battery are the same, since the final energy level is also the initial condition for the next day scheduling. Hence, the following equality constraint on the initial and final $S O C$ of batteries is enforced

$$
b_{s}^{0}=b_{s}^{T} .
$$

Moreover, the initial level should be high enough to allow a flexible use of the battery: in this study, we assume $b_{s}^{0}=$ $\underline{b}_{s}+\frac{\bar{b}_{s}-\underline{b}_{s}}{2}$. Now the proposed multi-objective optimization problem of jointly scheduling smart appliances and battery could be written as

$$
\begin{array}{cl}
\underset{\begin{array}{c}
\cos _{p, x, s, t, b_{s},}, b_{d}, x_{c}, x_{d}, \\
c_{t}, d_{t}, p_{G}
\end{array}}{\operatorname{minimize}} & \sum_{k=1}^{m}\left((1-\lambda) E^{k}+\lambda C^{k}\right) p_{G}^{k}, \quad \lambda \in[0,1] \\
\text { subject to } & \text { constraints (3), (4), (6)-(11), (13)-(20) } \\
& p_{i j}^{k} \in \mathbb{R}, \quad \forall i, j, k \\
& x_{i j}^{k}, s_{i j}^{k} \in 0,1, \quad \forall i, j, k \\
& t_{i j}^{k} \in 0,1, \quad \forall i, k, \quad \forall j=2, \ldots, n_{i} \\
& b_{s}^{k}, b_{c}^{k}, b_{d}^{k}, p_{G}^{k} \in \mathbb{R}, \quad \forall k \\
& x_{c}^{k}, x_{d}^{k}, c_{t}^{k}, d_{t}^{k} \in 0,1, \quad \forall k,
\end{array}
$$


which can be solved by CPLEX.

\section{Impact of DR signals on the automated active houses}

As it was discussed in previous sections, DR signals provide costumers an opportunity to save electricity bill and $\mathrm{CO}_{2}$ emission by shifting consumption and using batteries. To illustrate potential future benefits of automation systems in active houses which are provided by DR signals, 10000 apartments are considered as a case study. For this case study, three different scenarios including reference apartments (without automation system), test apartment (equipped with automation system), and test apartment with battery (equipped with automation system and battery) have been taken into account and compared with each other. Throughout the comparison, average hourly power consumption data from the mentioned two real active apartments (Fig. 3) is used, and is considered as the average hourly power consumption of the reference apartment, and the technical specifications of the smart appliances (dishwasher, washing machine, and dryer) have been extracted from [11]. For each scenario, the number and types of the smart appliances that are running in one day in those 10000 apartments, can be calculated from these technical specification, average hourly power consumption data from the two real active apartments in the day, and considering the fact that $4 \%$ of energy consumption is devoted to the washing-machine and dryer and $4 \%$ for dish-washer. Thus, by having the number and types of smart appliances, solving the multi-objective optimization (12) for scheduling of appliances yield to average hourly power consumption for the 10000 test apartments. To include batteries in the automation systems, one should consider some limitations (e.g. charging rate and capacity), inefficiencies, and nonlinear relationships between life cycles and depth of discharge (DOD). A battery with the following specifications is applied for the apartments with batteries

- Maximum DOD: $30 \%$

- Storage capacity: $1700(\mathrm{Wh})$

- Maximum power exchange: $1000(\mathrm{~W})$

- Stored energy degradation $(\alpha)$ : negligible

- Charging and discharging efficiency: $90 \%$

- Maximum charging and discharging cycles: 5 (per day). Solving the multi-objective optimization (21) for scheduling of appliances and battery (with the mentioned specifications), yields the average hourly power consumption for the 10000 test apartments with battery. In Fig. 4 average hourly power consumption curves related to smart appliances and battery, for these three scenarios of apartments for June 2013, is shown. Note that, to show the differences more clearly, only the consumption related to the smart appliances and batteries has shown. In addition, the total bill and $\mathrm{CO}_{2}$ savings in these 10000 apartments for the test apartment and test apartment with battery are compared in Table I, in terms of percent and amount of saving. In all the simulations, hourly price tariffs for June 2013 are downloaded from Nordpool website [22]. In addition, the SVK website [23] provide us with electricity generation by fuel type data, electricity import, and electricity export for 2013 and hourly $\mathrm{CO}_{2}$ foot print curves can be
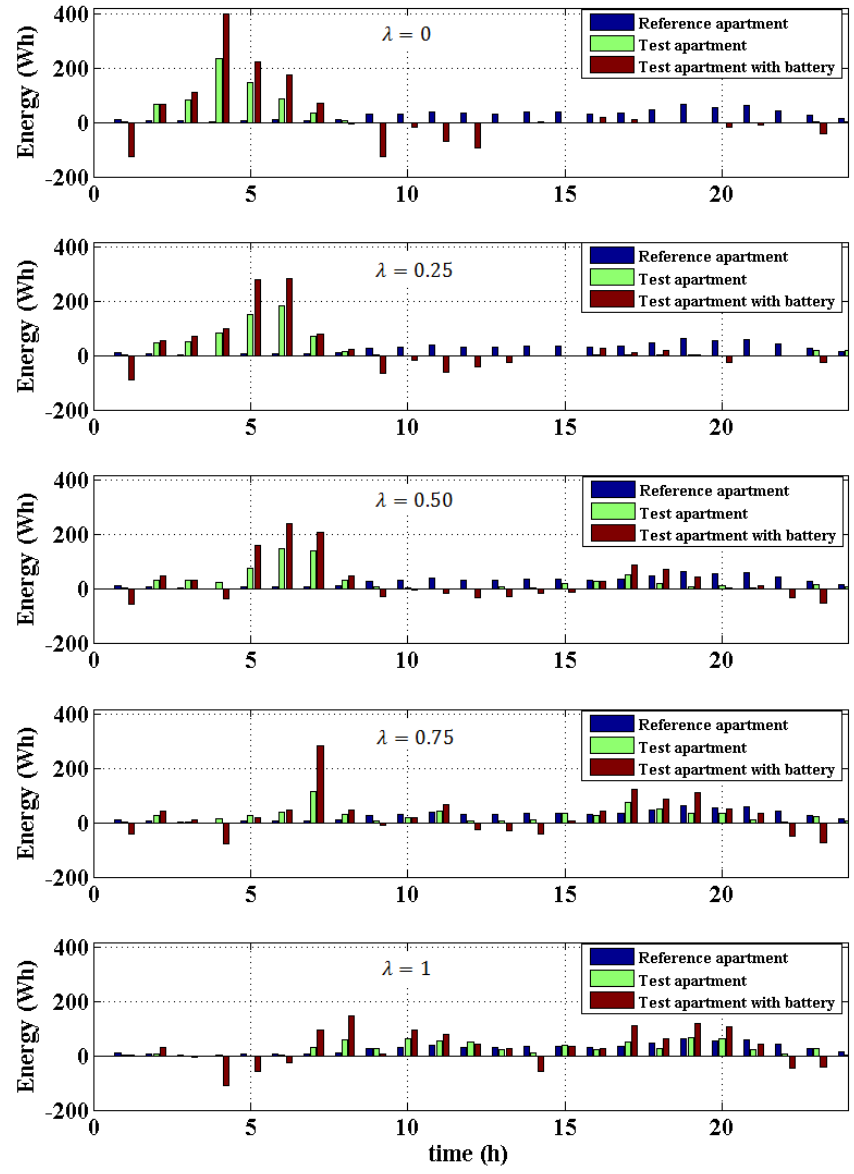

Fig. 4. Average hourly load curves of three type of active apartments in June 2013 for different values of the weight parameter $(\lambda)$.

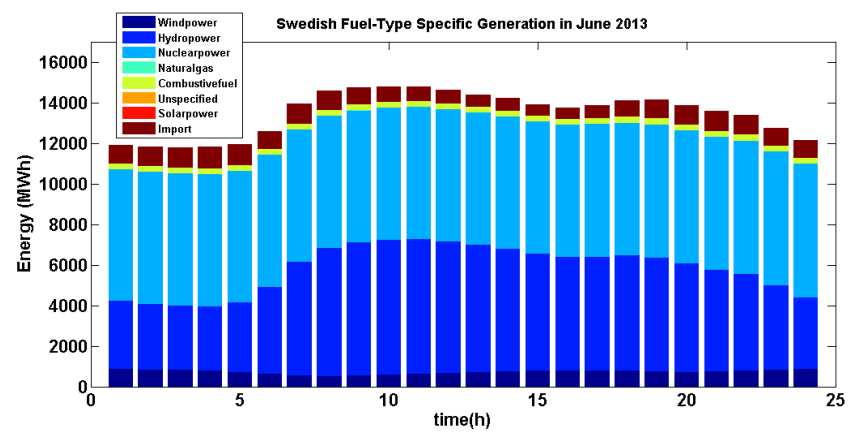

Fig. 5. Average hourly Swedish Fuel-Type Specific Generation in June 2013.

computed based on these data [10]. Average hourly electricity generation by fuel type data, import and export for June 2013 is shown in Fig. 5.

\section{Discussion}

1) Environmental and economic benefits: As it is mentioned in [10], in certain countries like Sweden there sometimes exists a trade-off between environmental and economic consideration, and for some electricity generation mixes, price and $\mathrm{CO}_{2}$ intensity are negatively correlated. Moreover, it is shown in [10] that the Swedish $\mathrm{CO}_{2}$ intensity is very sensitive to import of high carbon intensity power generation. Thus, considering only economic incentives for shifting load 
TABLE I

BILL AND $\mathrm{CO}_{2}$ SAVING IN 10000 ACTIVE APARTMENTS (JUNE 2013).

\begin{tabular}{|l||l|l|l|l|l|}
\hline$\lambda$ & 0 & 0.25 & 0.5 & 0.75 & 1 \\
\hline \multicolumn{7}{|c|}{ Saving without using battery } \\
\hline $\mathrm{CO}_{2}(\%)$ & -2.88 & -0.23 & 1.05 & 1.79 & 1.98 \\
\hline $\mathrm{CO}_{2}(\mathrm{~kg})$ & -2330 & -183 & 849 & 1447 & 1602 \\
\hline bill (\%) & 2.41 & 2.16 & 1.56 & 0.68 & -0.22 \\
\hline bill (SEK) & 19013 & 17032 & 12305 & 5336 & -1751 \\
\hline \multicolumn{7}{|c|}{ Saving by using battery } \\
\hline $\mathrm{CO}_{2}(\%)$ & -5.01 & 0.37 & 5.56 & 7.66 & 8.02 \\
\hline $\mathrm{CO}_{2}(\mathrm{~kg})$ & -4057 & 297 & 4501 & 6200 & 6491 \\
\hline bill (\%) & 4.94 & 4.10 & 2.46 & 0.70 & -1.10 \\
\hline bill (SEK) & 38948 & 32304 & 19371 & 5502 & -8669 \\
\hline
\end{tabular}

could result in an increased $\mathrm{CO}_{2}$ emission, and this is obvious in the case under study. Figure 4 shows the comparison between the average hourly energy profiles of smart home appliances and batteries for the reference apartment, test apartment, and test apartment with battery under different attitude of users toward the electricity bill and $\mathrm{CO}_{2}$ emission (different $\lambda$ ). Comparing Figures 4 and 5 we may notice that for $\lambda=0$ (considering economic profits only), the load tends to be shifted to the hours when the ratio of import energy to energy sources such as hydropower and nuclear power is higher (between 03:00 and 06:00), because Sweden imports are relatively inexpensive [12]. This leads to $4.94 \%$ bill saving for test apartments with batteries (Table I) that is more than twice the saving in test apartment without battery (2.41\%). This scenario yields $\mathrm{CO}_{2}$ emission to be increased with $2.88 \%$ and $5.01 \%$ for test apartments without and with batteries, respectively, which is not environmentally desired. This is due to the use of energy imported during night from Denmark, Germany and Poland whose primary energy source is combustive fuel power plants and is $\mathrm{CO}_{2}$ intense $(303,430$, and $640 \mathrm{gCO}_{2} / \mathrm{kWh}$ respectively), while clean energy sources such as hydropower and nuclear power have negligible $\mathrm{CO}_{2}$ intensity (4 and $16 \mathrm{gCO}_{2} / \mathrm{kWh}$ respectively) [10].

On the opposite, for $\lambda=1$ (caring about environmental impact only), the load tends to be distributed within the hours when the ratio of import energy to clean energy sources such as hydropower and nuclear power is lower (between 07:00 and 22:00). As it is illustrated in Table I, scheduling in test apartment with battery yields $8.02 \% \mathrm{CO}_{2}$ saving (more than four times of the saving in the test apartment without battery), while increasing the electricity expenses by $1.1 \%$, which is not desired economically. Therefore, for most customers it is most convenient to care both environmental and economic benefits. Consider, for instance, $\lambda=0.5$ and the test apartments with battery: in this case, we can have $5.56 \%$ and $2.46 \% \mathrm{CO}_{2}$ and bill saving, respectively, which is desirable both environmentally and economically.

2) Impacts of uncertainty in scheduling problem: The scheduling program in Subsection III-C provides scheduling within 24 hours to get the optimal solution, which is the most optimistic scenario and difficult to be achieved in practice because consumers can change their usage patterns during the day. This can be represented in the proposed scheduling framework by considering different time preference intervals.
TABLE II

BILL AND $\mathrm{CO}_{2}$ SAVING IN 10000 ACTIVE APARTMENTS, AFFECTED BY TIME PREFERENCES (JUNE 2013)

\begin{tabular}{|l||l|l|l|l|l|}
\hline$\lambda$ & 0 & 0.25 & 0.5 & 0.75 & 1 \\
\hline \multicolumn{7}{|c|}{ Saving without using battery } \\
\hline $\mathrm{CO}_{2}(\%)$ & -1.31 & 0.71 & 1.59 & 1.80 & 1.84 \\
\hline bill (\%) & 0.62 & 0.42 & 0.14 & -0.09 & -0.41 \\
\hline \multicolumn{7}{|c|}{ Saving by using battery } \\
\hline $\mathrm{CO}_{2}(\%)$ & -3.44 & 1.31 & 6.10 & 7.67 & 8.02 \\
\hline bill (\%) & 3.15 & 2.36 & 1.04 & -0.07 & -1.39 \\
\hline
\end{tabular}

Hence, to investigate the impact of user time preferences on the load shift, the scheduling of appliances for the three types of apartment (reference, test, and test with battery) have been computed under different $\lambda$ and between 08:00 and 24:00 hours. Note that this time interval has been chosen based on Figure 3, which shows that families in active apartments are more interested to run their appliances within this period. Bill and $\mathrm{CO}_{2}$ savings in the 10000 apartments for the test apartment and test apartment with battery scenarios are compared in Table II. As it is obvious in this Table, the percentage of $\mathrm{CO}_{2}$ saving for $\lambda=0.75$ and $\lambda=1$ are approximately the same (for both test apartment and test apartment with battery scenarios), while for smaller $\lambda$ this saving increases, which is reasonable. In this period, energy generation from clean energy sources such as hydropower is higher and $\mathrm{CO}_{2}$ emission is less, so by choosing the mentioned time preference interval, loads could not to be shifted to the early day hours (for example in the case of $\lambda=0$ ) when $\mathrm{CO}_{2}$ is intense. But bill saving has drastically decreased for different $\lambda$, and both types of apartments. Instead of the case $\lambda=1$, this reduction is larger for the apartments without batteries. For example in the case $\lambda=0$, for the apartment without battery bill saving decrease from $2.41 \%$ to $0.62 \%$ which means $75 \%$ reduction in saving, while the same case for the apartment with battery causes to a reduction of less than $40 \%$ in saving.

3) Battery profitability: To take the cost of batteries into account, and to investigate whether the battery usage in the proposed method is profitable or not, life cycles of the deployed battery is calculated. In the literature, lead-acid batteries for smart houses are commonly utilized [24]. In the manufacturers battery specification data sheet for the leadacid batteries, the number of life cycles versus different DOD is given. Further, charging and discharging efficiencies for lead-acid batteries are generally $85-95 \%$. If we consider the schedule of smart appliances and batteries in the test apartments in June 2013 (for example for $\lambda=0.25$ ), simulation results show that for 10000 apartment in 30 days, batteries will be used 475513 times in total, and the DOD is $21 \%$ on average. Based on the mentioned data sheet, for this percent of DOD, the number of life cycles is 2600 for a battery. We can conclude that a battery would be economically viable if it costs less than 2500 SEK. However, we remark that the use of battery is environmentally beneficial. 


\section{PERFormanCE OF DIFFERENT METHODS FOR SCHEDULING HOME APPLIANCES}

As it was mentioned before, based on our knowledge until now, the MILP framework in [11] is more extendable than the others for scheduling of smart appliances. But for being solved, it has some limitation in computational time and it is valuable to compare it with the other formulations. In addition to MILP framework in [11], there are two other problem formulations and methods (by using time discretization) for scheduling smart home appliances.

Dynamic programming (DP): A more general case is considered in [12] where the electricity tariff and $\mathrm{CO}_{2}$ footprint signals (demand response (DR) signals) are arbitrary. In that paper, a dynamic programming based procedure is applied to solve the multi-objective scheduling problem, in a test case with drastically different electricity price and $\mathrm{CO}_{2}$ emission patterns. As the two objective are in conflict with each other, the $\varepsilon$-constraint approach is considered for exploration and trade-off analysis through Pareto curve, but it is obvious that weighted sum approach could be used in the same procedure. Minimum cut algorithm (MCA): In [13], the same setting as in [12] is used for scheduling of appliances, user preferences and technical operation constraints. Then a discrete time formulation for the problem is proposed and is solved by finding a minimum-cut in a suitably defined diagraph, while a weighted sum approach is considered for multi-objective scheduling problem. It is shown in the paper that this method results in a near-optimal cost and reduces computational time.

For comparing these three frameworks for solving the scheduling problem, simulations are all done on a 64bit Windows system with an Intel Core i7-3770, 3.40GHz and 16.0 GB of RAM.

\section{A. Computation time and optimality versus time discretiza- tion}

As it was considered in [13], we also use an instance introduced and solved in [12] to compare the solving time and optimal cost of the three mentioned methods, as a function of time discretization. For this comparison, scheduling of three appliances including washing machine, dryer, and dishwasher is studied and all the appliances specifications, user preferences, and the hourly price tariff are detailed in [13]. This instance has been solved by the three formulations for values of discretization time $\left(N_{t}\right)$ which are the number of time slots in a day, ranging from 24.15 to 24.65 by steps of 5 . The computation time and optimal cost of this simulation is shown in the Fig. 6. As it is obvious in left of this figure, the computation time of MILP is more than two orders of magnitude larger than MCA and DP. Moreover, as it is pictured in the right of Fig. 6, MILP yields to a more accurate solution for the optimization problem for all the discretization resolution that is around $0.56 \%$ on average, and the two other methods results in the same cost as each other. In addition, it shows that by increasing the discretization from $24 \cdot 15$ to $24 \cdot 65$, MILP can improve the
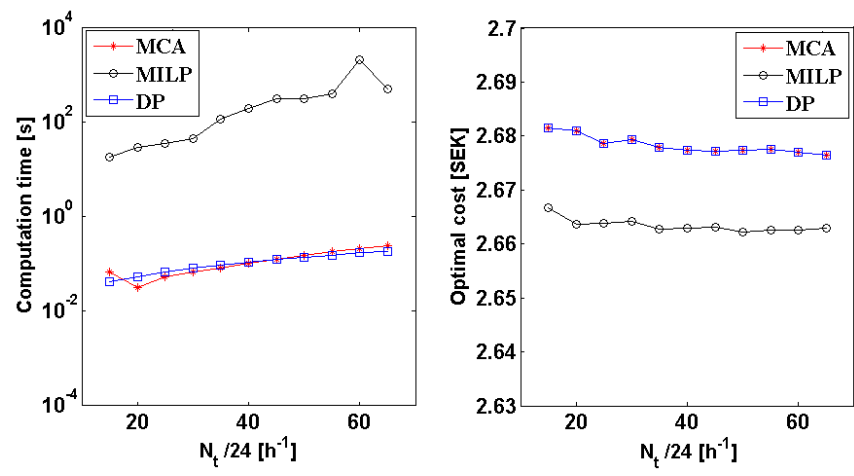

Fig. 6. Computation time and optimality versus discretization.

optimal cost by $0.15 \%$ which is negligible, and increasing the resolution is not worth computational burden. Therefore, as discretization increases, DP and MCA perform much better than MILP in computational time while having a negligible error in minimum cost computation compare to the MILP.

\section{B. Computation time and optimality versus number of appli-} ances

To compare the solving time and optimality of the solution for the growing number of appliances, a set of three appliances with 5 energy phases (e.p.'s) is considered, and we increase the number of appliances by adding one of that set at each step. As it is mentioned in this paper, in the MILP formulation the information related to the upper and lower limits on the power assignment of each energy phases, and also the limits on energy phases process time is required, so it can not be chosen randomly (unlike the case discussed in [13]). In this paper, these three appliances have been defined based on the specification of washing machine and dishwasher that are detailed in [13], means the first appliance has the same specifications as the first 5 e.p.'s of dishwasher, the second appliance has the same specification as the first 5 e.p.'s of washing machine, and the third one has the specifications that are twice of the specifications of the first one. The time is discretized in $N_{t}=24.6$ (every 10 minutes) and the simulation results are shown in Fig. 7. As it is obvious in the right of Fig. 7, they all have approximately the same optimal cost as long as the number of appliances increases. In addition, it is pictured in left of Fig. 7, that MILP results in a computational time more than three orders of magnitude larger, and also the computational time of MCA is always less than DP. Regarding the implementation of these methods, it should be mentioned that MCA has been implemented in $\mathrm{C}++$ and is used in Matlab through a mex-file, while the others are implemented in Matlab, and this is one of the reasons that make MCA to have much less computational time in comparison with the others. The other point is that, the DP implementation in [12], is based on $\varepsilon$-constraint while here it is implemented based on weighted sum cost function, and it is much faster (about 200 times) than the one in [12].

\section{Conclusion and Future Studies}

This paper proposes a new method for scheduling smart home appliances and batteries. A novel optimal scheduling 

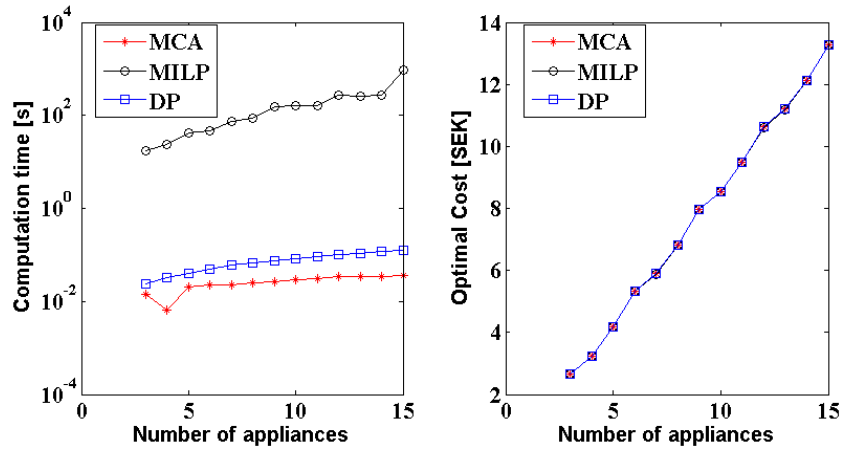

Fig. 7. Computation time and optimality versus number of appliances.

formulation is posed as a multi-objective Mixed Integer Linear Programming (MILP), which not only decreases the $\mathrm{CO}_{2}$ emissions and the electricity bill, but also takes the state of health of batteries into account. A realistic case study of 10000 active apartments in the Royal Seaport project was investigated for assessing the impacts of DR signals on load shifting and bill and $\mathrm{CO}_{2}$ saving factors. This assessment is based on different attitudes of users toward the environmentally and economic benefits (different $\lambda$ ). It has been shown that, the more consumers care about the electricity price, the more $\mathrm{CO}_{2}$ emission is produced (for $\lambda$ close to one). An optimized use of the battery can further increase daily cost saving and $\mathrm{CO}_{2}$ emission reduction. Further, a comparison of related papers that apply DP and MCA for scheduling of smart appliances is given, and their performance in terms of computation time and optimality versus time discretization and number of appliances is discussed. It shows that solution time for the scheduling problem formulated as a MILP increases exponentially when the number of appliances increases, and also when the discretization resolution increases, but it is much easier to include batteries in the MILP formulation. Uncertainties related to the scheduling of appliances, have also been studied in terms of time preferences, and as a future study the other uncertainties such as interrupting the appliances, or customary scheduling of appliances, could be analysed. In addition, as it was discussed, only $8 \%$ of power consumption of the active apartments is devoted to the smart appliances, and almost half of it is related to the lightning, heating and cold appliances. Thus, by taking these consumptions into account in automated systems, the bill and $\mathrm{CO}_{2}$ savings could be significantly increased.

\section{REFERENCES}

[1] D. O’Neill, M. Levorato, A. Goldsmith, and U. Mitra, "Residential demand response using reinforcement learning," in A report to the United Stated Congress pursuant to Section 1252 of the energy policy act of 2005, 2006.

[2] S. Singh and J. Ostergaard, "Use of demand response in electricity markets: An overview and key issues," in Energy Market (EEM), 2010 7th International Conference on the European, 2010, pp. 1-6.

[3] J. M. Lujano-Rojas, C. Monteiro, R. Dufo-Lopez, and J. L. BernalAgustin, "Optimum residential load management strategy for real time pricing (rtp) demand response programs," Energy Policy, vol. 45, pp. 671-679, 2012.

[4] S. Gottwalt, W. Ketter, C. Block, J. Collins, and C. Weinhardt, "Demand side management a simulation of household behavior under variable prices," Energy Policy, vol. 39, pp. 8163-8174, 2011.
[5] C. Bartusch, F. Wallin, M. Odlare, I. Vassileva, and L. Wester, "Introducing a demand-based electricity distribution tariff in the residential sector: Demand response and customer perception," Energy Policy, vol. 39, pp. 5008-5025, 2011.

[6] Z. Xu, J. Ostergaard, and M. Togeby, "Demand as frequency controlled reserve," Power Systems, IEEE Transactions on, vol. 26, pp. 10621071, 2011.

[7] J.-H. Kim and A. Shcherbakova, "Common failures of demand response," Energy, vol. 36, pp. 873-880, 2011.

[8] N. G. Dlamini and F. Cromieres, "Implementing peak load reduction algorithms for household electrical appliances," Energy Policy, vol. 44, pp. 280-290, 2012.

[9] M. Song, K. Alvehag, J. Widen, and A. Parisio, "Estimating the impacts of demand response by simulating household behaviours under price and co2 signals," in Electric Power Systems Research, 2014, pp. 40514058.

[10] P. Stoll, N. Brandt, and L. Nordstrom, "Including dynamic $\mathrm{CO}_{2}$ intensity with demand response," Energy Policy, vol. 65, pp. 490 $500,2014$.

[11] K. C. Sou, J. Weimer, H. Sandberg, and K. H. Johansson, "Scheduling smart home appliances using mixed integer linear programming," in Decision and Control and European Control Conference (CDC-ECC), 50th IEEE Conference on, 2011, pp. 5144-5149.

[12] K. C. Sou, M. Kordel, J. Wu, H. Sandberg, and K. H. Johansson, "Energy and $\mathrm{CO}_{2}$ efficient scheduling of smart home appliances," in Control Conference (ECC), European, 2013, pp. 4051-4058.

[13] J. M. Hendrickx, R. M. Jungers, G. Vankeerberghen, and L. A. Wolsey, "An efficient technique for solving the scheduling of appliances in smart-homes," in American Control Conference (ACC), June 2014, pp. 4051-4058.

[14] J. P. Zimmermann, "End-user metering campaign in 400 households in Sweden, assessment of the potential electricity savings," in ENERTECH, Tech. Rep., 2009, pp. 4051-4058.

[15] P. Yi, X. Dong, A. Iwayemi, C. Zhou, and S. Li, "Real-time opportunistic scheduling for residential demand response," Smart Grid, IEEE Transactions on, vol. 4, pp. 227-234, 2013.

[16] [Online]. Available: http://www.stockholmroyalseaport.com/en/

[17] X. Chen, T. Wei, and S. Hu, "Uncertainty-aware household appliance scheduling considering dynamic electricity pricing in smart home," Smart Grid, IEEE Transactions on, vol. 4, pp. 932-941, 2013.

[18] Y. Riffonneau, S. Bacha, F. Barruel, and S. Ploix, "Optimal power flow management for grid connected pv systems with batteries," Sustainable Energy, IEEE Transactions on, vol. 2, pp. 309-320, 2011.

[19] J. Lofberg, "Yalmip : a toolbox for modeling and optimization in matlab," in Computer Aided Control Systems Design, IEEE International Symposium on, 2004, pp. 284-289.

[20] S. Boyd and L. Vandenberghe, Convex Optimization. Cambridge University Press, 2004.

[21] A. Parisio, C. Del Vecchio, and A. Vaccaro, "A robust optimization approach to energy hub management," in International Journal of Electrical Power \& Energy Systems, 2012.

[22] [Online]. Available: http://www.nordpoolspot.com/

[23] [Online]. Available: http://www.svk.se/

[24] A. Mishra, D. Irwin, P. Shenoy, J. Kurose, and T. Zhu, "Smartcharge: Cutting the electricity bill in smart homes with energy storage," pp. $1-10,2012$. 